\title{
Management of Complicatd Crown Root Fracture - A Case Report
}

Srikanth Raju S , Rajender Reddy E², Thabitha Rani S³, Kiranmayi M4, Srujana MP5

Quick Response Code

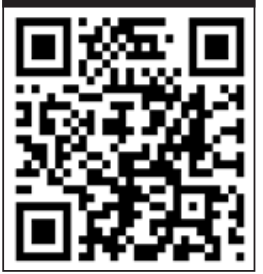

doi: $10.5866 / 2017.9 .10106$

IPost Graduate Student

2Prof \& HOD

${ }^{3}$ Professor

${ }^{4}$ Reader

${ }^{5}$ Senior Lecturer

Department of Pedodontics

Kamineni Institute of Dental Sciences,

Sreepuram, Narketpally, Nalgonda (Dt).

\section{Article Info:}

Received: April 11, 2017

Review Completed: May 13, 2017

Accepted: J une 12, 2017

Available Online: J une, 2017 (www.nacd.in)

(c) NAD, 2017 - All rights reserved

Email for correspondence:

srikanth1090@gmail.com

\section{ABSTRACT:}

Functional, esthetic and biologic restoration of a fractured incisor often presents a daunting clinical challenge. This case report describes the surgical, endodontic, prosthetic and restorative treatment of complicated crown root fracture of maxillary central incisor.

Key words: compl icated crown fracture, sub gingival, fiber post.

\section{INTRODUCTION}

A permanent tooth suffering from trauma could be a considerable problem especially for young patients owing to functional and esthetic causes. A crown-root fracture is a type of dental trauma, usually resulting from horizontal impact, which involves enamel, dentin and cementum, occurring below the gingival margin. These can becategorized as complicated or uncomplicated, depending on the pulp involvement. ${ }^{1}$ The incidence of complicated crown root fractures ranges from $2 \%$ to $13 \%$ of all dental injuries. ${ }^{2}$
Several factors influence the management of coronal tooth fractures, including extent of fracture (biologic width violation, endodontic involvement, alveolar bone fracture and restorability of fractured tooth) secondary trauma injuries (soft tissuestatus), presence /absence of fractured tooth fragment and its condition for use (fit between fragment and the remaining tooth structure),occlusion, aesthetics, finances and prognosis. ${ }^{3}$ The objective of the present paper is to describe the multi- disciplinary approach of complicated crown root fractures involving endodontic, prosthetic, surgical and restorative procedures. 


\section{CASE REPORT}

A thirteen year old female patient reported with a chief complaint of fractured upper anterior teeth. She gave a history of fall 6 months back with a missing fragment of fractured upper front tooth. I mmediately after trauma patient had visited nearby dentist, who prescribed medication for relieving pain.

Clinical examination revealed presence of Ellis class III fracture in maxillary right central incisor (11) and a complicated crown root fracture in maxillary left central incisor (21) (Figure 1). In maxillary left central incisor the fracture line enrooted from the center of incisal edgethrough the pulp chamber extending beyond the cervical line into the sub gingival area. Patient had no other soft tissue lesions. No mobility was observed in both the teeth. Radiographic examination revealed distinct periapical radiolucency involving both the maxillary left and right central incisors (Figure 2). One important complication in the treatment planning was the sub gingival extension of fractured margin.

Non-surgical approach was initially planned with the use of calcium hydroxide and iodoform (metapex). Treatment was initiated with access cavity preparation in 11 and 21; necrotic pulp tissue from the root canals was extirpated and canal was irrigated with physiological saline and dried with paper points. Working length was established $1 \mathrm{~mm}$ short of apex. Bio mechanical preparation was done with $\mathrm{k}$ file up to size 40 . The canal was once again irrigated with physiological saline and then dried with paper points. Root canals of both 11 and 21 were then filled with Calcium hydroxide and iodoform (metapex) beyond the apex with the intention to resolve peri apical abscess and the access cavity was restored with temporary restorative material (Figure 3). Follow up of patient was done for 8 months, within which there was no resolution of peri apical lesion was seen.

It was then decided to opt periapical surgery for the treatment of lesion following the preliminary blood investigations. The root canals were irrigated properly with $5.25 \%$ sodium hypochloriteand finally obturated with gutta-percha and zinc oxide eugenol sealer with the lateral condensation technique (Figure 4). The area was anesthetized and a full thickness mucoperiosteal flap was raised on the buccal side. A bony defect was evident in the apical region of the both the central incisors (Figure 5). The bony defect was curetted properly and irrigated with normal saline. Bonegraft followed by PRF were placed in bony defect and suturing was done with non resorbable suture material (silk). The patient was recalled after 7 days for suture removal. At the same visit post space preparation was done in 21 . Gutta-percha within the canal was removed up to 4 $\mathrm{mm}$ short of obturated length. A suitable size fiber reinforced post was inserted into the prepared canal. Fractured area of tooth that lies sub-gingival was replaced with composite resin to prevent in growth of gingiva into fractured region followed by cementation of fiber post with GIC. Aesthetically matched composite was used to reconstruct the fractured crown of 21 using free hand technique and composite core build up done in 11. Crown preparation done in 21 after 3 days and PFM crown cemented in the next visit (Figure 6). Clinical and radiographic examinations were carried out at 3 and 6 months follow up. Resolution of peri apical radiolucency was seen and patient was satisfied both esthetically and functionally with the outcome of treatment.

\section{DISCUSSION:}

Dental traumas due to crown-root fracture are rarely seen when compared to crown fractures (Marceneset al., 1999; Bastoneet al., 2000). It is even rare to see a complicated crown -root fracture. There are few treatment options available in treating complicated crown root fractures: (Brown and Welbury 2000; Fariniuket al., 2003).

- Removal of the fractured coronal fragment and restoration of tooth if the fracture line has not encroached into the biologic width

- Removal of the coronal fragment and supplemented with gingivectomy and osteotomy to expose the fracture in order to establish biologic width prior to restoration

- Removal of the coronal fragment and initiation of endodontic treatment and restoration of tooth with post crown

- Removal of the coronal fragment and initiation of endodontic treatment and later by orthodontic or surgical extrusion of the 


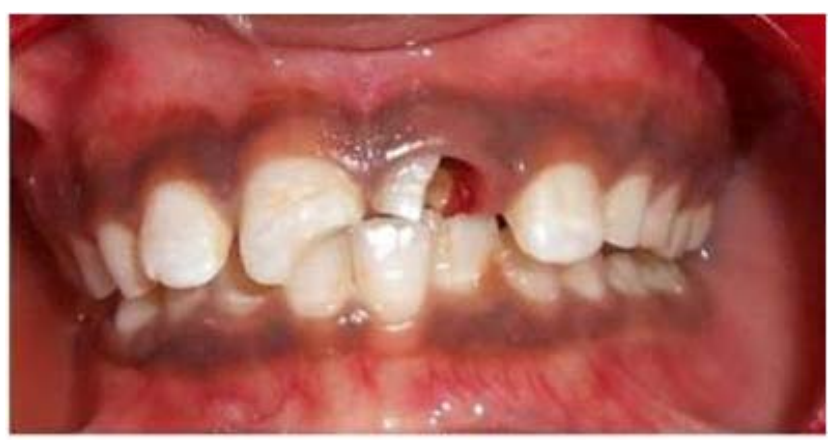

Figure 1: Clinical picture showing presence of Ellis class III fracture in maxillary right central incisor and a complicated crown root fracture in maxillary left central incisor.

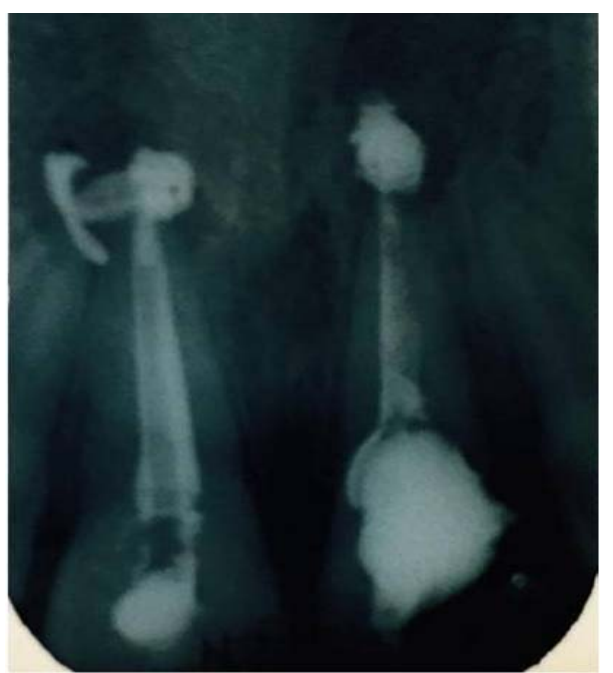

Figure 2: IOPA shows distinct periapical radiolucency involving both the maxillary left and right central incisors

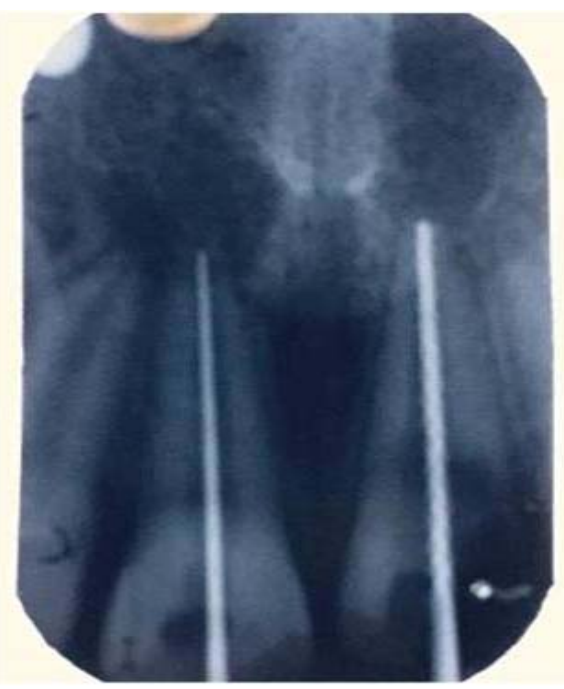

Figure 3: IOPA showing Root canals of 11 and 21 filled with Calcium hydroxide and iodoform (metapex) beyond the apex with the intention to resolve peri apical abscess and the access cavity was restored with temporary restorative material

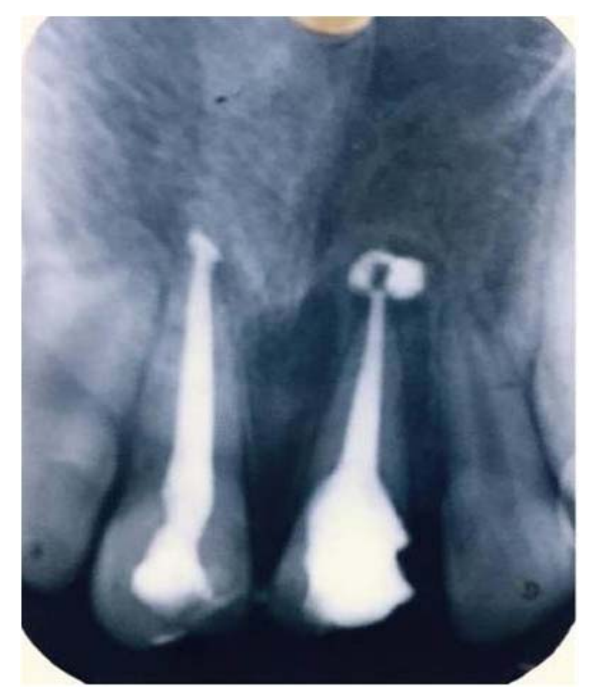

Figure 4: IOPA radiograph showing obturation of 11 and 21 with gutta-percha and zinc oxide eugenol sealer with the lateral condensation technique

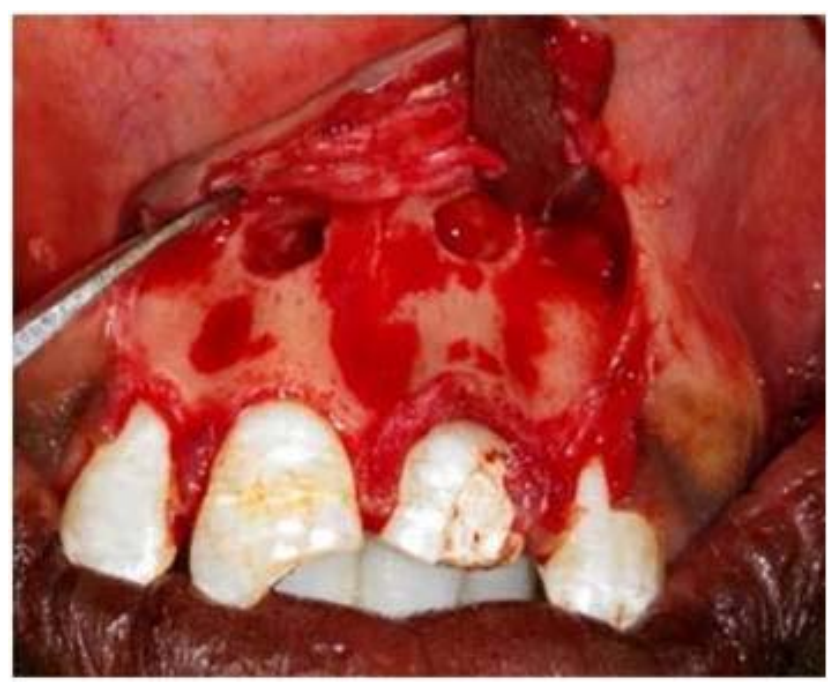

Figure 5: Evidence of bony defect in the apical region of the both the central incisors which is curetted followed by bone grafting and placement of PRF.

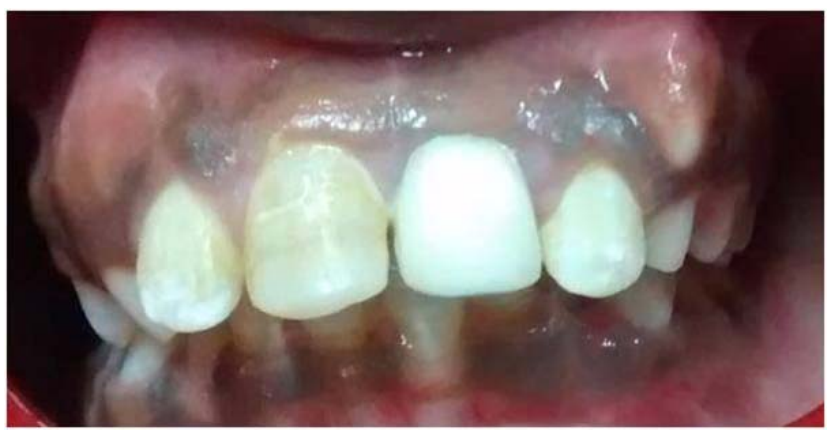

Figure 6: Cementation of PFM crown. 
apical fragment prior to restoration with post crown.

- In severe crown - root fracture, the tooth may have to be extracted and replaced with removal or fixed prosthesis4.

In the present case initially effort was made to treat the case conservatively through a nonsurgical approach. It was only when the nonsurgical method failed, the surgical procedure was performed to treat peri apical abscess. Bone graft and PRF were placed into the defect for faster enhancement of lesion.

To reinforce the cervical level of the reattached tooth, it is recommended to use an intracanal post since these interlock the coronal and root fragments and also minimizethe stress on the reattached tooth fragment. $^{5-7}$ It has been suggested that the use of a long, thin fiber post is effective for reducing tensile stress that can lead to tooth root fractures of the anterior teeth with endodontic treatments. ${ }^{8}$ In the present case, the fracture line was extending subgingivally and the tooth was pulpless. Hence, it was decided to gain intraradicular retention by using fiber posts.

In the present case, there were some possible options for the treatment: extraction should not be an option of treatment because it leads to loss of bone in the anterior area, compromising future treatment with eventual implants. Moreover, placement of implant during childhood is contraindicated before the end of the alveolar growth. Orthodontic extrusion is an alternative for the treatment of sub-gingival fractures. Nevertheless this alternative affects esthetics and occlusion and requires longer time considering the period of extrusion and stabilization. ${ }^{9,}{ }^{10}$ When intact fragments are available, reattachment would be the best option of the treatment, it may offer many advantages: better esthetics, less time consuming and positive emotional and social response from the patient for the preservation of natural tooth structure but the limitation of this case report was the patient missed the fractured fragment. ${ }^{11}$ As fractured fragment was not there the sub gingival fractured area was replaced with composite upto free gingival margin. Currently the patient was under annual follow up.

\section{CONCLUSION}

The treatment strategy of complicated crownroot fracture is complex, due to sub-gingival position of the fracture margin encroached on the biological width. The present clinical report demonstrated the need of a multidisciplinary intervention during the treatment of complicated crown root fracture. I nitially calcium hydroxide was used to resolve the lesion, but as the lesion was not resolved peri apical surgery was performed in 21 . Fiber post was cemented in 21 to reinforce the cervical level of restoration. PFM crown preparation doneirt 21 and later PFM crown cemented in 21. Patient was followed up to 6 months. Further follow up was needed to evaluate the success of treatment.

\section{References}

1. Andreasen J O, Andreasen FM. Textbook and color atlas of traumatic injuries to the teeth. 4th ed. Oxford: Blackwell; 2007.

2. Geeta Preethi S. Managment of complex crown root fracture using fibre post- a case report. Endodontology 2014; 26 (1):211-6.

3. Shikha J aiswal, Sachin Gupta, Abhishek Gupta. Slaving a Subgingivally Fractured Maxillary Lateral Incisor: A multidisciplinary approach http://www.Iivedental.in/articles/ endodontics.

4. Nagarajan S, Sockalingam MP, Alida Mahyuddin. Complicated crown root fracture treatment option -a case report. Archives of Orofacial Sciences 2009; 4(1):25-8.

5. Kumar A and K. N. J yothi, "Reattachment of fractured tooth using self-etching adhesive and esthetic fiber post,"J Dental Sci Res 2010; 1:75-83.

6. Wangand J, Li M. "Multidisciplinar treatment of a complicated crown-root fracture," Pediatric Dent 2010; 32(3):250- 4.

7. Tosun G, Yildiz E, Elbay M and Sener Y. "Reattachment of fractured maxillary incisors using fiber-reinforced post: two case reports,". Eur J Dent 2012; 6(2):227-33.

8. Nakamura T, Ohyama T, Waki T et al. "Stress analysis of endodontically treated anterior teeth restored with different types of post material,". Dental Materials J 2006; 25(1):14550.

9. Kudou Y, Kubota M. Replantation with intentional rotation of a complete vertically fractured root using adhesive resin cement. Dent Traumatol 2003; 19:115-7.

10. Emerich PK, Sawicki L, Bodal M, Adamowicz KB. Forced eruption after crown/root fracture with a simple and aesthetic method using the fractured crown. Dent Traumatol 2005; 21:165-9.

11. Fawzi R, Hariri M. The Treatment Strategy of an Oblique Complicated Crown-root Fracture: Case Report. Pediatr Dent Care 2016; 1: 110. 